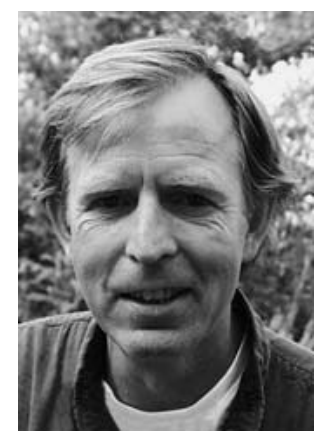

\title{
Celebrating LINKS - The Homeopathic Conference
}

Next year is the 20th anniversary of LINKS. To celebrate this we have organised an international homeopathic conference to which you are all cordially invited.

Putting Vision into Practice

The aim of the conference is to showcase the evolution of classical homeopathy in the past two decades, an evolution which has been largely influenced by new ideas discussed in LINKS, and to project these into the future. Massimo Mangialavori, Rajan Sankaran and Jan Scholten have been invited as the key speakers since they have contributed considerably to this development, used LINKS as a platform, and are still actively expanding the horizons of our art and science.

The key speakers will each give a morning seminar in which the focus will be on the main development they went through over the years, what have been the observations, theoretical considerations and experiences that have led them to the point where they are now, what they are presently working on now, and how they perceive the future development of homeopathy.

\section{Meet and Link!}

By letting one kingdom and one key speaker set the themes for each day of the conference we intend to structure an interactive process of integration during the conference. This will be strongly stimulated by several other speakers invited for each day, who will give a short overview on how they have applied the work of the key speakers and other developments in their practice. In this way we hope to make visible how the different developments can be integrated and are being extended.

Programme

Friday October 192007 the Mineral Kingdom will be the main topic. Besides a seminar by Jan Scholten the following speakers will in two parallel sessions contribute to this day: Jayesh Shah, Jorge Loukas, Jeremy Sherr, Louis Klein, Alize Timmerman and Andreas Björndal.

On Saturday October 20 the Plant Kingdom will be in the spotlight. Following a seminar by Rajan Sankaran, presentations in parallel sessions will be made by Resie Moonen, Linda Johnston, Patricia Leroux, Irene Schlingensiepen-Brysch, Will Taylor and Uta Santos.

On Sunday October 21 Massimo Mangialavori will take the lead regarding the Animal Kingdom, followed by Annette Sneevliet and Anne Schadde.

Since there are more kingdoms besides the three main ones from which homeopathy gratefully receives its remedies Frans Vermeulen will give a presentation on Friday on Monera and Fungi.

\section{Integration}

We would like the conference to be a dynamic experience for all participants. We have therefore invited all speakers to stay for the whole conference and to participate in the discussions. At the end of the conference Jeremy Sherr together with the key speakers will wind up the proceedings.

With the combined synergy of these varied contributions from skilled and experienced colleagues we feel this can indeed become the conference every homeopath would want to attend.

By distilling what have been the most useful developments over the past years, by showing where we are able to find common ground and where we are able to weave the different strands into a fabric of good results in our practice, and by projecting these into the future, we intend and expect that together we can envision the future development of homeopathy.

\section{Organisation}

Celebrating LINKS - The Homeopathic Conference will take place at a most beautiful venue in Heidelberg, Germany, at October 19-21, 2007. Sonntag Verlag, the publisher of LINKS, will organise the conference with the participation of the European Council of Classical Homeopathy (ECCH) and the European Committee for Homeopathy (ECH). The organising committee consists of the following persons: Stephen Gordon (ECCH), Ton Nicolai (ECH), Gabriele Müller and Sverre Klemp (Publisher), Caroline Augspurger-Hacker (Conference office), Corrie Hiwat (Cultural programme) \& Harry van der Zee (Editor).

I look forward to meeting you all during October 19-21 2007 in Heidelberg.

For more details please see the announcement printed in this issue and visit the conference website at http:// www.homeopathic-conference.de.

On behalf of the organising committee, Harry van der Zee, editor 